Revista Motricidad Humana / Julio - Diciembre, Edición 13(2)

\title{
DISTRIBUCIÓN DE PRESIONES MUÑÓN-PRÓTESIS Y TEMPERATURA SUPERFICIAL DEL MUÑON EN AMPUTADOS TRANSTIBIALES
}

\section{PRESSURE DISTRIBUTION STUMP-PROSTHESIS AND SUPERFICIAL TEMPERATURE OF THE STUMP IN TRANSTIBIAL AMPUTEE}

\author{
Berral Aguilar, Francisco de Borja ${ }^{1}$; Ezquerro Juanco, Francisco ${ }^{1}$; Sosa Tallei, Guillermo Oscar ${ }^{2}$, Berral de la \\ Rosa, Francisco José ${ }^{2}$
}

\author{
${ }^{1}$ Departamento de Ingeniería Mecánica y Mecánica de Fluidos. Escuela Técnica Superior de Ingenieros Industriales. Universidad de \\ Málaga. España \\ ${ }^{2}$ Departamento de Deporte e Informática. Universidad Pablo de Olavide. Sevilla. España
}

BERRAL A.F.B., EZQUERRO J.F., SOSA T.G.O., BERRAL D.L.R.F.J. Distribución de Presiones Muñón-Prótesis y Temperatura Superficial del Muñón en Amputados Transtibiales. Mot.Hum. 13(2): 84-92; 2012.

\section{RESUMEN}

El presente estudio pretende analizar la distribución de las presiones que ejerce el muñón del amputado transtibial en el interior del encaje protésico y observar si existe alguna correlación con el aumento de la temperatura superficial del mismo. Del mismo modo se pretende diseñar un protocolo de estudio para el análisis de la relación entre estas variables que influyen en la comodidad y calidad de vida del paciente amputado.

Hemos valorado cinco pacientes de edad media de 35 años que llevaban amputados al menos 10 años, con un peso medio $85 \mathrm{~kg}$ y estatura de $1,77 \mathrm{~m}$.

Para el estudio de distribución de presiones, se ha utilizado el sistema K-Scan de TEKSCAN y para la temperatura una cámara termográfica marca FLIR, modelo T360.

No encontramos relación entre los puntos de presión y los valores de gradiente de temperatura. No existe relación entre la distribución del $\Delta T$ (incremento de temperatura) en el muñón producida tras la marcha y la distribución de presiones medida durante la misma. Por tanto no parece que el nivel de presiones entre el muñón y la prótesis sea el causante de los $\Delta T$ observados en los muñones de los amputados transtibiales.

Palabras clave: amputados, termografía, distribución de presiones

\begin{abstract}
This study aims to analyze the distribution of the pressures exerted by the transtibial amputee stump inside the prosthetic socket and see if there is any correlation with increasing surface temperature itself. The same is intended to design a study protocol for the analysis of the relationship between these variables that influence the comfort and quality of life of patients amputees.

We evaluated five patients of mean age 35 who had amputees at least 10 years, with an average weight of $85 \mathrm{~kg}$ and height of $1.77 \mathrm{~m}$. To study the pressure distribution, the system has been used was K-Scan of TEKSCAN and for temperature a thermal camera FLIR brand, model $T 360$.

We can't found relationship between the pressure points and the values of temperature gradient. No relationship exists between the distribution of $\Delta T$ (temperature increase) on the stump produced after the march and the pressure distribution measured during the same. Therefore it seems that the level of pressures between the stump and the prosthesis isn't causing the observed $\Delta T$ in the stumps of transtibial amputees.
\end{abstract}

Key Words: amputee, thermography, pressure distribution. 
BERRAL A.F.B., EZQUERRO J.F., SOSA T.G.O., BERRAL D.L.R.F.J. Distribución de Presiones Muñón-Prótesis y Temperatura Superficial del Muñón en Amputados Transtibiales. Mot.Hum. 13(2): 84-92; 2012.

\section{INTRODUCCIÓN}

Valores medidos sobre presiones del encaje protésico han sido reportados por Laing y col en 2011 (1). Una investigación que nos ayudó a elegir los puntos de medida de presión en el encaje protésico fue la realizada por Dou y col en 2006 (2).

Para identificar las zonas donde se midieron las presiones con los sensores en la imagen termográfica, se utilizaron marcadores de aluminio debido a su baja emisividad. Esta metodología se obtuvo del estudio realizado por Howell y col en 2009 (3).

Para Kristen y col en 1984 (4) es muy importante identificar las zonas de medición de temperatura y poder compararlas, cuestión que hemos tenido en cuenta en nuestro estudio.

De los distintos aspectos que intervienen en la calidad de vida de los amputados transtibiales, tal vez el más importante sea la comodidad y adaptabilidad del encaje protésico en el muñón, lo que sin duda permite una mejor funcionalidad. Esta preocupación ha sido lo que ha motivado a Ali y col en 2012 (5) a llevar a cabo una investigación en 9 amputados transtibiales. Dicho estudio ha sido de referencia para nosotros en cuanto a los estandares de confortabilidad de los amputados.

El objetivo principal del presente estudio es analizar la distribución de las presiones que ejerce el muñón del amputado transtibial en el interior del encaje protésico y observar si existe alguna correlación con el aumento de la temperatura superficial del mismo en las zonas de mayor presión. Del mismo modo vamos a diseñar un protocolo de estudio para el análisis de la relación entre estas variables fundamentales que influyen en la comodidad y calidad de vida del paciente amputado, e intentaremos obtener una base de datos de valores de presión y temperatura del muñón, antes y después del ciclo de marcha, tanto de la cara anterior como posterior de la extremidad del muñón de pacientes amputados transtibiales.

\section{MATERIAL Y METODOS}

Hemos valorado cinco pacientes amputados transtibiales de una edad media de 35 años que llevaban amputados al menos 10 años, con un peso medio de $85 \pm 6 \mathrm{~kg}$ y estatura 1'77 \pm 0 0'04 m.

Para la medida de las presiones hemos usado una plantilla deformable. El sensor, modelo 5076, que hemos utilizado pertenece al sistema K-Scan. El mismo posee una caja de conexiones que hace de "traductor" entre el software y el sensor. El software denominado I-Scan permite visualizar en tiempo real el mapa de presiones que se está generando y grabar los datos para su tratamiento analítico.

Todos los sensores utilizados se han calibrado siguiendo las recomendaciones del fabricante, mediante una máquina (figura 1) dotada con una célula de carga marca HBM modelo U2B (Darmstadt, Germany).

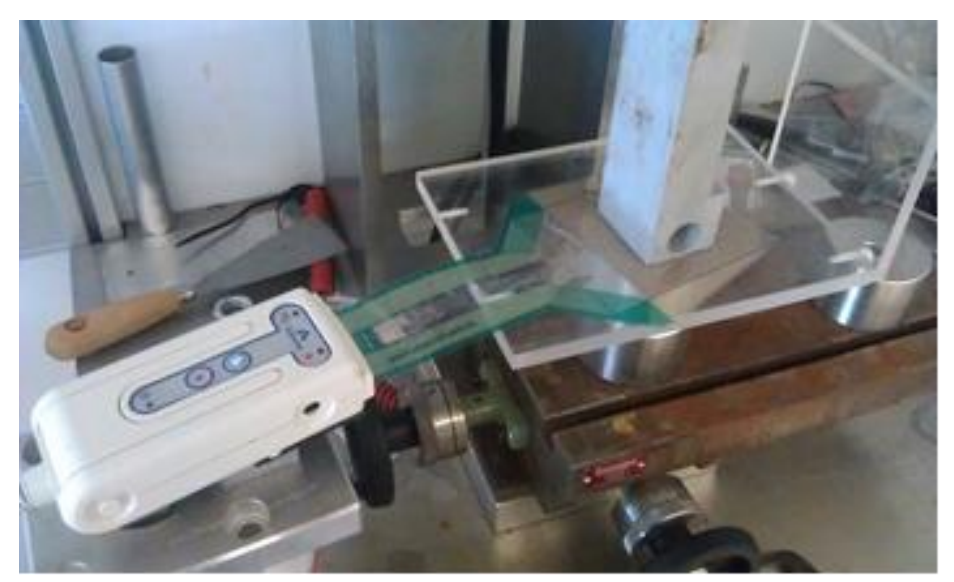

Figura 1: Calibrado del sensor de medición de presiones 
Los sensores se acondicionan a una presión del $80 \%$ de la carga máxima que se quiera medir. Posteriormente se utiliza el software (I-Scan) para la calibración, que se realiza mediante dos puntos, uno de ellos al $80 \%$ de la carga máxima esperable y otro al $20 \%$. La calibración se realiza para varias sensibilidades, normalmente entre 36 y 38 unidades, siendo 40 el máximo admitido por los sensores y el software.

La temperatura ha sido medida con una cámara termográfica marca FLIR, modelo T360 Western, que posee un sensor que capta la luz infrarroja, que es analizada por otro sensor electrónico que procesa la imagen.

El software del ordenador, FLIR QuickReport, permite realizar el análisis de la imagen mediante herramientas propias, los valores numéricos obtenidos de temperatura pertenecientes a cada pixel han sido exportados, tratados y analizados con el programa informático Microsoft Excel, el cual también hemos utilizado para el estudio de los valores de presión.

\section{Diseño del protocolo.}

Nada más llegar el paciente al laboratorio se le hace caminar por los pasillos a ritmo ligero durante 10 minutos.

Posteriormente se quita la prótesis y de forma inmediata se toman imágenes térmicas del muñón (Figura 2), tanto de la cara anterior como posterior, con el fin de apreciar la temperatura del muñón después de realizar una actividad física cotidiana, en este caso caminar.

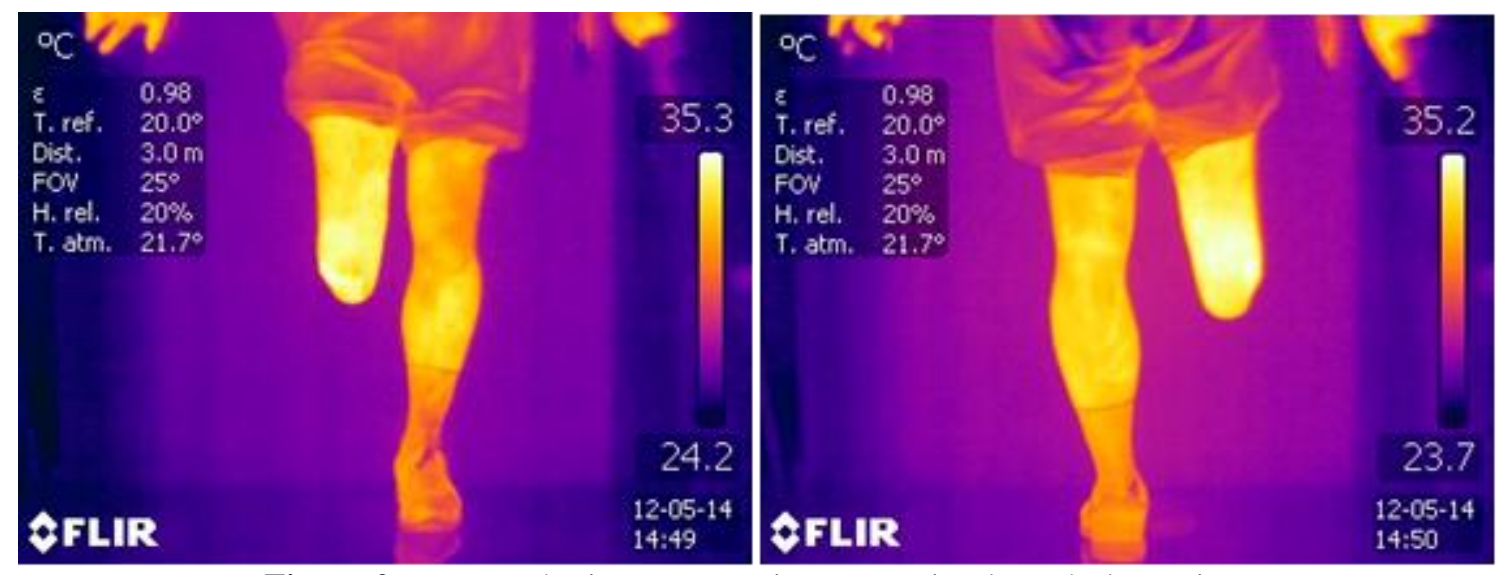

Figura 2: Imagen térmica cara anterior y posterior después de caminar

Precauciones a tener en cuenta:

- Las luces y las ventanas del laboratorio tienen que estar cerradas para que la captación de luz infrarroja no influya en la medida.

- El paciente debe de estar alejado lo máximo posible de superficies que tengan una emisividad alta, debido a que actúan de espejo térmico.

- La posición del trípode y del paciente deben permanecer invariables entre las distintas medidas realizadas.

- El papel de plata colocado en el sensor como marcador de su posición se intentará tocar lo menos posible, debido a que esté absorbe calor rápidamente al ser manipulado.

- La temperatura ambiente del laboratorio debe estar en torno a $25^{\circ} \mathrm{C}$ para que todas las fotografías térmicas se tomen en las mismas condiciones ambientales.

Una vez obtenidas las imágenes, el paciente descansa en una silla con el muñón libre, para que éste se estabilice y el riego sanguíneo sea lo más natural posible. Al cabo de 15 minutos se le realizan dos fotografías térmicas iguales a las anteriores a fin de comparar las diferencias de temperatura.A continuación se coloca una plantilla de presiones en la zona anterior del muñón (Figura 3) y otra en la zona posterior. 


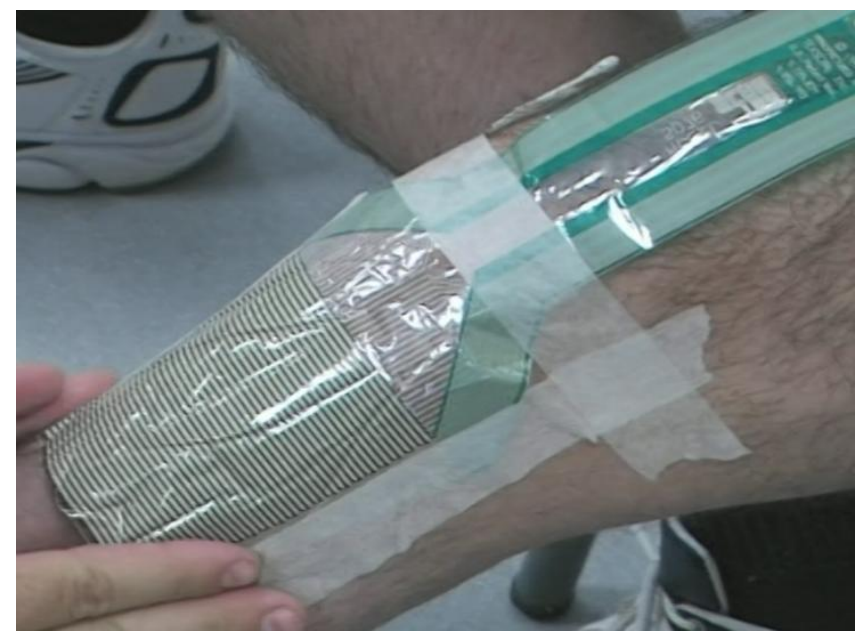

Figura 3: Colocación de plantilla anterior en el muñón

La colocación es importante de cara a la posterior inserción de la silicona, y que esta no arrugue mucho el sensor, para así poder tomar mediciones de la presión en la marcha con la mayor precisión.

Antes de que el paciente se coloque la silicona se vuelve a realizar una fotografía térmica, tanto de la cara anterior como posterior, con la diferencia de que ésta se realiza con papel de aluminio en la zona donde está el sensor de presión $(3,6)$.

Se utiliza papel de aluminio porque su "emisividad" ( $\varepsilon$ ) es muy baja, del orden de $0,05 \mathrm{~mm}$, mientras que la emisividad del cuerpo humano es bastante alta, del orden de 0,98. De esta manera identificamos a posteriori la zona de medición del sensor de presiones.
Para la reproducibilidad del experimento se utilizan marcas en el suelo y pared, además de un trípode.

A continuación se conectan las plantillas de presión a la caja de conexiones, procediendose a un calibrado de las mismas, esto es debido a que al ser tan sensibles, por el simple hecho de estar doblada, provoca que se detecten valores de presión, los cuales hay que eliminar.

El siguiente paso es dejar que el paciente se coloque la silicona sobre el muñón y lo encaje en la prótesis (Figura 4).

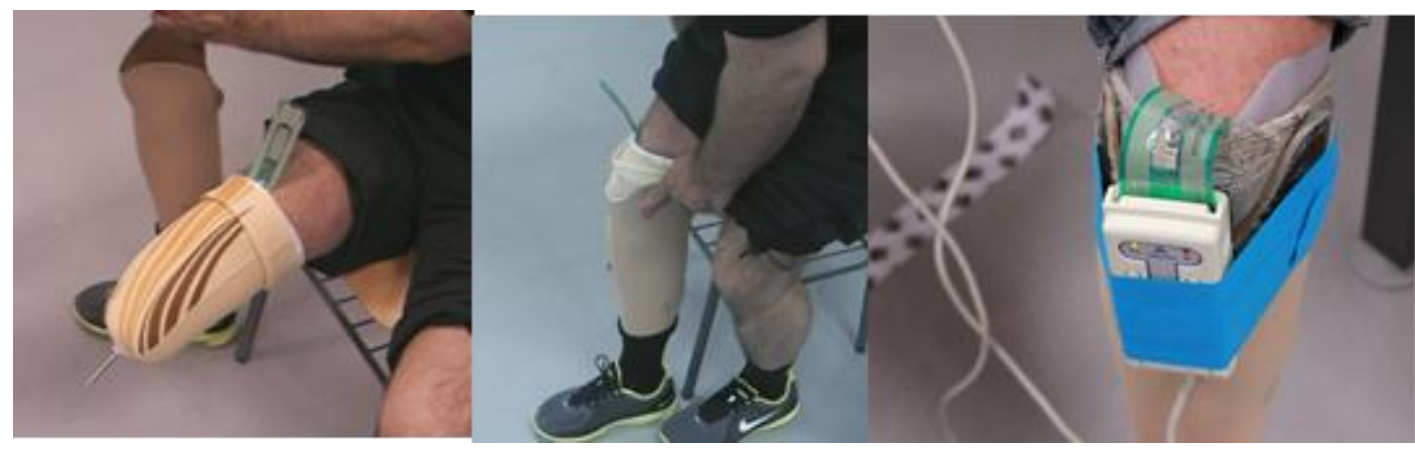

Figura 4: Silicona con sensor en cara anterior, colocación de la prótesis y caja de conexión

Hay que comprobar que el paciente pueda caminar correctamente, sin molestias, tras lo cual se procede a conectar la caja de conexiones (Figura 4). Se hace andar al paciente en línea recta, es suficiente con que la persona camine unos 10 o 12 pasos ya que nos centraremos en un solo ciclo de marcha. 
BERRAL A.F.B., EZQUERRO J.F., SOSA T.G.O., BERRAL D.L.R.F.J. Distribución de Presiones Muñón-Prótesis y Temperatura Superficial del Muñón en Amputados Transtibiales. Mot.Hum. 13(2): 84-92; 2012.

Realizamos una grabación de toda la secuencia del ciclo de marcha, para la posterior identificación de las fases del mismo. A continuación conectamos el sensor posterior y llevamos a cabo el mismo protocolo.

Los datos de presión de cada frame grabado provienen de la exportación desde el programa IScan. En nuestro caso hemos guardado datos con una frecuencia de $1 / 20$. De este programa exportamos dos grupos de datos, los datos pertenecientes a la presión en cada frame, con una resolución de 44 x 44 puntos, que son en los que está dividido el sensor. Estos datos se organizan mediante un código en el programa informático Microsoft Excel en matrices de 44 x 44, subdivididas de la siguiente forma si el paciente tiene amputada la pierna derecha (Tabla I y Figura $5)$.

\begin{tabular}{ccc}
\hline Matriz proximal lateral. & Matriz proximal central. & Matriz proximal medial. \\
Tamaño $15 \times 15$. & Tamaño $15 \times 14$. & Tamaño $15 \times 15$. \\
\hline Matriz medial lateral. & Matriz central. & Matriz central medial. \\
Tamaño $14 \times 15$. & Tamaño $14 \times 14$. & Tamaño $14 \times 15$. \\
\hline Matriz distal lateral. & Matriz distal central. & Matriz distal medial. \\
Tamaño $15 \times 15$. & Tamaño $15 \times 14$. & Tamaño $15 \times 15$. \\
\hline
\end{tabular}

Tabla I: Divisiones de la matriz de presiones.

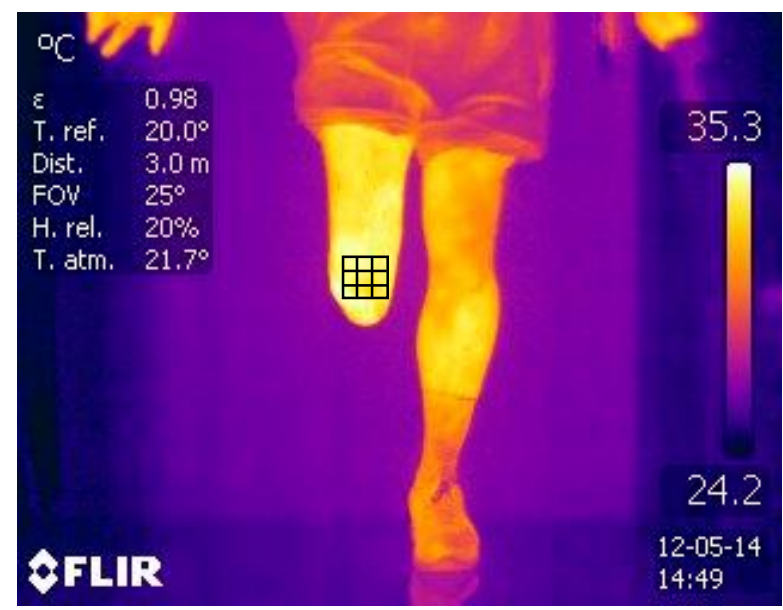

Figura 5: Visualización de las distintas zonas de análisis de las matrices

A estas submatrices le realizamos la media, obteniendo una matriz de 3 × 3 con los valores promedio de cada submatriz. Esta matriz promedio es la que utilizamos para el análisis de las presiones, sin olvidarnos de los valores pico de presión.

Mediante el coeficiente de correlación de Pearson se estudió la linealidad de los dos parámetros principales, la temperatura y la presión en el muñón. Este coeficiente ha sido aplicado en el estudio 1 y 2 de los 3 realizados.
En el estudio 3 se utilizó el porcentaje del incremento de temperatura en el muñón $(\% \Delta \mathrm{T})$ en la zona donde se registró la presión pico. Esto sirvió para ver qué porcentaje de incremento de temperatura se producía en las zonas donde la presión ejercida era máxima.

\section{RESULTADOS}

Respecto a la presión, mediante un código de programación en Microsoft Excel se tratan los datos de todos los frames obteniendo las matrices, submatrices y matrices promedio de cada individuo, sin saber aún que intervalo o ciclo de marcha vamos 
BERRAL A.F.B., EZQUERRO J.F., SOSA T.G.O., BERRAL D.L.R.F.J. Distribución de Presiones Muñón-Prótesis y Temperatura Superficial del Muñón en Amputados Transtibiales. Mot.Hum. 13(2): 84-92; 2012.

a escoger. Para ello exportamos los datos de las gráficas de Fuerza versus Tiempo y Pico de presión versus Tiempo desde el programa I-Scan.

Estos datos se vuelven a tratar con Microsoft Excel mediante un programa que se ha elaborado para tal fin, obteniendo automáticamente las gráficas. Una vez obtenidas y con la ayuda de la grabación de vídeo que hemos realizado del ciclo de marcha, identificamos un ciclo de marcha intermedio el cual será el que analicemos.

Escogido éste, procedemos a extraer las matrices promedio de los frames que corresponden a ese ciclo, lo cual también se realiza mediante un código de programación. Finalizado este proceso, obtenemos los datos de presión para analizar junto a sus gráficas correspondientes, para después buscar la relación entre la presión máxima en un ciclo de marcha y la zona donde se encuentre el mayor incremento de temperatura. motivo los resultados corresponden a las diferencias encontradas entre la temperatura en el muñón tras el reposo y justo después de caminar. Estos valores se han medido en la zona del muñón donde está colocado el sensor, para así poder comparar las presiones con los incrementos térmicos producidos durante la marcha (Figura 6).

Los valores característicos que acompañan al mapa de temperaturas son:

- Valor promedio de la diferencia de temperatura antes y después de andar.

- Diferencia máxima registrada entre el valor de la temperatura antes y después de caminar.

- Diferencia mínima registrada entre el valor de la temperatura antes y después de caminar.

En relación a este último parámetro, interesa saber si ha habido o no gradiente de temperatura, donde se ha producido y cuál ha sido su valor. Por este

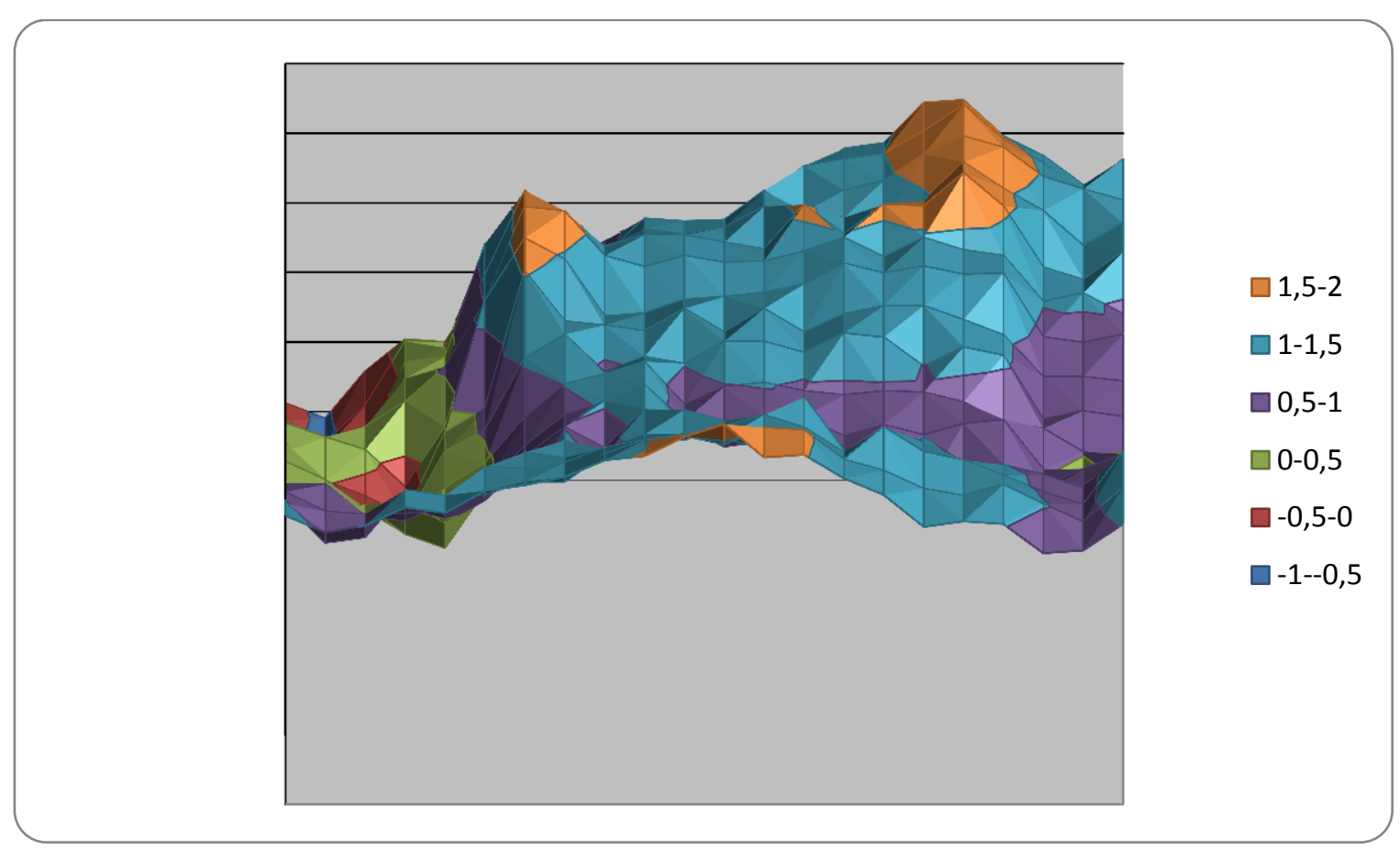

Figura 6: Representación en 3D del mapa de $\Delta \mathrm{T}$, cara anterior. Paciente 5 


\section{Estudio 1. Presión media máxima versus $\Delta \mathrm{T}$ medio}

Para este estudio hemos utilizado las submatrices medias de incrementos de temperatura y submatrices medias de presión en el instante de presión máxima (Tabla II).

\begin{tabular}{ccc}
\hline $\begin{array}{c}\text { Coef. Pearson } \\
\text { cara anterior }\end{array}$ & & $\begin{array}{c}\text { Coef. Pearson } \\
\text { cara posterior }\end{array}$ \\
\hline$-0,48$ & Paciente 1 & 0,10 \\
0,05 & Paciente 2 & $-0,30$ \\
0,16 & Paciente 3 & $\mathbf{0 , 9 0}$ \\
$-0,30$ & Paciente 4 & 0,43 \\
0,60 & Paciente 5 & $-0,32$ \\
\hline
\end{tabular}

Tabla II: Coeficientes de Pearson para el estudio 1

Podemos observar que valores no son constantes, incluso cambian de signo de positivo a negativo, interpretando que su relación pueda ser directa o inversa. Podemos decir que no se han encontrado unos valores que nos indiquen que existe alguna relación lineal ni directa ni inversa entre los resultados estudiados. Sólo en el paciente 3 en la cara posterior se ha encontrado un valor de éste coeficiente cercano a 1 el cual nos indica que existe una relación lineal, pero dados los valores de los otros pacientes habría que descartar con más pruebas este resultado. Añadir que no se aprecia que los resultados obtenidos sigan otro tipo de tendencia entre ellos, ya sea exponencial o logarítmica.

\section{Estudio 2. Presión media del ciclo versus $\Delta T$ medio}

Aquí utilizamos las submatrices de presión media a lo largo del ciclo, de esta forma obtenemos una visión global de la relación entre el $\Delta \mathrm{T}$ y la presión media soportada a lo largo del ciclo de la marcha (Tabla III). Este estudio es interesante de cara a que las zonas de mayor presión media a lo largo del ciclo no son las mismas que las zonas donde se soporta la mayor presión pico.

\begin{tabular}{ccc}
\hline $\begin{array}{c}\text { Coef. Pearson } \\
\text { cara anterior }\end{array}$ & Coef. Pearson & $\begin{array}{c}\text { Cora posterior } \\
\text { cara }\end{array}$ \\
\hline$-0,52$ & Paciente 1 & 0,12 \\
0,18 & Paciente 2 & $-0,16$ \\
$-0,17$ & Paciente 3 & $\mathbf{0 , 8 7}$ \\
$-0,22$ & Paciente 4 & 0,25 \\
0,63 & Paciente 5 & 0,04 \\
\hline
\end{tabular}

Tabla III: Coeficientes de Pearson para el estudio 2

Al igual que con anterioridad, los valores del coeficiente de Pearson nos indican que no existe relación lineal, sólo en el paciente 3 en la cara posterior se observa que existe relación lineal, pero como hemos comentado puede ser un resultado que nos confunda, por lo tanto tendría que contrastarse con más pruebas y medidas.

Estudio 3. \% $\% \mathrm{~T}$ producido en las zonas donde se soportan las presiones pico máximas.

En este estudio se cuantifica si existe relación o no entre los datos de presión pico de cada individuo y el incremento de temperatura medio, pero utilizando el valor porcentual de éste en la misma zona donde se encuentra el valor pico en la matriz de presiones.

Para calcular el $\% \Delta \mathrm{T}$ se ha utilizado la fórmula siguiente en cada zona de la submatriz media de temperaturas:

$$
=\frac{\text { Elemento a analizar - valor mínimo de la matriz }}{\text { valor máximo de la matriz - valor mínimo de la matriz }}
$$

Los valores se muestran en las tablas IV y V.

\begin{tabular}{lcc}
\hline & P pico max & \% $\mathbf{\Delta T}$ \\
\hline Paciente 1 & 281,00 & 81,24 \\
Paciente 2 & 359,00 & 37,13 \\
Paciente 3 & 191,00 & 69,71 \\
Paciente 4 & 189,00 & 56,82 \\
Paciente 5 & 354,00 & 80,23 \\
\hline
\end{tabular}

Tabla IV: Valores de la cara anterior 
BERRAL A.F.B., EZQUERRO J.F., SOSA T.G.O., BERRAL D.L.R.F.J. Distribución de Presiones Muñón-Prótesis y Temperatura Superficial del Muñón en Amputados Transtibiales. Mot.Hum. 13(2): 84-92; 2012.

\begin{tabular}{lcc}
\hline & P pico max & \% $\Delta \mathbf{T}$ \\
\hline Paciente 1 & 178,00 & 81,56 \\
Paciente 2 & 150,00 & 79,19 \\
Paciente 3 & 141,00 & 78,10 \\
Paciente 4 & 103,00 & 81,66 \\
Paciente 5 & 91,00 & 56,49 \\
\hline
\end{tabular}

Tabla V: Valores de la cara posterior

Se puede observar que para la cara anterior no existe una relación entre el $\% \Delta \mathrm{T}$ sufrido y el valor de la presión pico máxima. De existir relación deberíamos obtener valores más o menos iguales para cada paciente de $\% \Delta \mathrm{T}$, y en este caso no ha sido así, hay valores muy dispares, desde el 81,24 \% hasta un $37,13 \%$.

En la cara posterior parece que hay un indicio de que existe alguna relación ya que los valores están en torno al $80 \%$, sólo hay un paciente que se sale de este rango.

\section{DISCUSION}

No hemos encontrado en la literatura artículos que hablen acerca de la relación entre estas dos magnitudes, la presión en el muñón y la temperatura tras la marcha.

Peery y col en 2005 (7) estudian, en 5 amputados, la comodidad de los pacientes según el material y tipo de la funda del muñón y del encaje. Obsevan que el muñón incrementa su temperatura una media de $0.8^{\circ} \mathrm{C}$ en reposo, y de $1.7^{\circ} \mathrm{C}$ tras la marcha. En nuestro estudio hemos observado incrementos de temperatura de ese orden de magnitud.

Estos incrementos de temperatura pueden ser debidos a la conductividad térmica del material de las fundas como ha estudiado Klute y col en 2007 (8), que encuentras valores de conductividad térmica muy dispares.

Tonon da Luz y col 2010 (9) observan temperaturas altas de los muñones con valores puntuales de $32,78^{\circ} \mathrm{C}$. Los autores opinan que estos datos pueden estar relacionados con la fricción dentro del encaje protésico lo que podría dificultar la marcha del paciente. Compartimos la opinión de estos autores, aunque no estamos de acuerdo de que sólo la adaptación del encaje protésico sea el factor determinante de los aumentos de temperatura.

Se ha observado que los pacientes que realizaban el ciclo de marcha correctamente tenían un solo punto de presión claramente marcado, mientras que los pacientes que realizaban el ciclo de marcha de manera incorrecta, es decir, cojeando o no realizando un movimiento armónico de la marcha, se obtenían dos puntos de presión.

Para amputados transtibiales, Zaproudina y col 2008 (10) y Harden y col 2008 (11) miden valores del miembro amputado y miembro sano para compararlas entre sí, y ver si se producen diferencias entre la temperatura de ambos. Los valores para miembros amputados en nuestro estudio están en el mismo rango de temperatura superfical que los aportados por estos autores.

El resultado obtenido de la investigación es satisfactorio desde el punto de vista de que no se tenía conocimiento de ningún estudio de este tipo, por lo tanto las conclusiones obtenidas y el protocolo diseñado esperamos ayuden a próximas investigaciones.

\section{CONCLUSION}

Para el grupo de pacientes estudiados podemos afirmar que no existe relación entre los puntos de presión y los valores de gradiente de temperatura.

No hemos encontrado relación entre la distribución del $\Delta \mathrm{T}$ en el muñón producida tras la marcha y la distribución de presiones medida durante la misma. Por tanto no parece que el nivel de presiones entre el muñón y la prótesis sea el causante de los $\Delta \mathrm{T}$ observados en los muñones de los amputados. 
BERRAL A.F.B., EZQUERRO J.F., SOSA T.G.O., BERRAL D.L.R.F.J. Distribución de Presiones Muñón-Prótesis y Temperatura Superficial del Muñón en Amputados Transtibiales. Mot.Hum. 13(2): 84-92; 2012.

\section{REFERENCIAS BIBLIOGRÁFICAS}

1. Laing S, Lee Pvs, Goh Jch. Engineering a trans-tibial prosthetic socket for the lower limb amputee. Ann Acad Med Singapore 2011; 40: 252.

2. Dou P, Jia X, Suo S, Wang R, Zhang M. Pressure distribution at the stump/socket interface in transtibial amputees during walking on stairs, slope and non-flat road. Clin Biomech 2006; 21: 1067-73.

3. Howell Kj, Lavorato A, Visentin Mt, Smith Re, Schaefer G, Jones Cd, Weibel L, Denton Cp, Harper Ji, Woo P. Validation of a protocol for the assessment of skin temperature and blood flow in childhood localized scleroderma. Skin Res Technol 2009; 15: 346-56.

4. Kristen H, Lukeschitsch G, Plattner F, Sigmund R, Resch P. Thermography as a means for quantitative assessment of stump and phantom pains. Prosthet Orthot Int 1984; 8: 76-81.

5. Ali S, Osman Na, Mortaza N, Eshraghi A, Gholizadeh H, Wan Abas Wa. Clinical investigation of the interface pressure in the trans-tibial socket with Dermo and Seal-In X5 liner during walking and their effect on patient satisfaction. Clin Biomech 2012; 27(9): 943-8.

$6 . \quad$ http://www.nivelatermografia.net/blog/tecnicas-demedicion-termografica-midiendo-la-emisividad/, (en línea enero 2012).

7. Peery Jt, Ledoux Wr, Klute Gk. Residual-limb skin temperature in transtibial sockets. J Rehabil Res Dev 2005; 42: 147-54.

8. Kluteg K, Rowe Gi, Mamishev Av, Ledoux Wr. The thermal conductivity of prosthetic sockets and liners. . Prosthet Orthot Int 2007; 32: 292-9.

9. Tonon Da Luz Sc, Avila Aov, Oliveira Tp, Andrade Mc, Ventoza Lacunza C, Berral De La Rosa Fj. Valoración del daño corporal en amputados de miembros inferiores: prueba de sensibilidad, postura, sobrecarga articular y calidad de vida. Trauma Fund MAPFRE 2010; 21: 178-83.

10. Zaproudina N, Varmavuo V, Airaksinen O, Närhi M. Reproducibility of infrared thermography measurements in healthy individuals. Physiol Meas 2008; 29: 515-24.

11. Harden Rn, Gagnon Cm, Gallizzi M, Khan As, Newman D. Residual Limbs of Amputees Are Significantly Cooler than Contralateral Intact Limbs. Pain Pract 2008; 8: 342-7.

\section{Dirigir Correspondencia a:}

Prof. Dr. Francisco José Berral de la Rosa. fjberde@upo.es

Carretera de Utrera km 1. 41013-Sevilla (España). Telf. y fax: 0034650139022 y 0034954348377 . e-

RECIBIDO: 16-10-2012

ACEPTADO: 26-10-2012 